\title{
Laser assisted solid state reaction for the synthesis of ZnS and CdS nanoparticles from metal xanthate
}

\author{
Damian C. Onwudiwe ${ }^{1}$, Tjaart P. Krüger ${ }^{2}$, Christien A. Strydom* ${ }^{1}$ \\ ${ }^{1}$ Chemical Resource Beneficiation (CRB) Research Focus Area, North-West University, Private Bag X6001, \\ Potchefstroom2520, South Africa \\ ${ }^{2}$ Department of Physics, University of Pretoria, Private Bag X20, Hatfield 0028, South Africa
}

\author{
* Corresponding author: Prof. Christien A. Strydom \\ Telephone: +27 182992340 \\ Fax: $\quad+27182992350$ \\ E-mail: christien.strydom@nwu.ac.za
}

\begin{abstract}
Nanoparticles of CdS and ZnS were produced by a nanosecond laser using zinc (II) and cadmium (II) complexes of ethyl xanthate. Laser pulses with a peak wavelength of $355 \mathrm{~nm}$, pulse repetition rate of $10 \mathrm{~Hz}$, and pulse duration of $\sim 4$ ns were used. The sample exposure times were $10 \mathrm{~min}$ and $30 \mathrm{~min}$ respectively. The obtained nanoparticles were characterized by scanning electron microscopy (SEM), transmission electron microscopy (TEM), ultraviolet-visible spectroscopy (UV-vis) and photoluminescence (PL) spectroscopy. The morphology and optical property of the synthesized nanoparticles were investigated as a function of the time of exposure. Upon extensive irradiation, the crystallinity of the CdS nanoparticles increased while the crystallinity of the ZnS nanoparticles decreased. The average crystallite size of the CdS nanoparticles estimated from the TEM image was $4.8 \mathrm{~nm}$, while the presence of aggregates with no crystalline edges impeded the size determination
\end{abstract}


of the ZnS nanoparticles. The absorption spectra showed that the nanoparticles exhibit quantum confinement.

Keywords: Nanosecond laser; CdS nanoparticles; ZnS nanoparticles; morphology

\section{Graphical abstract}
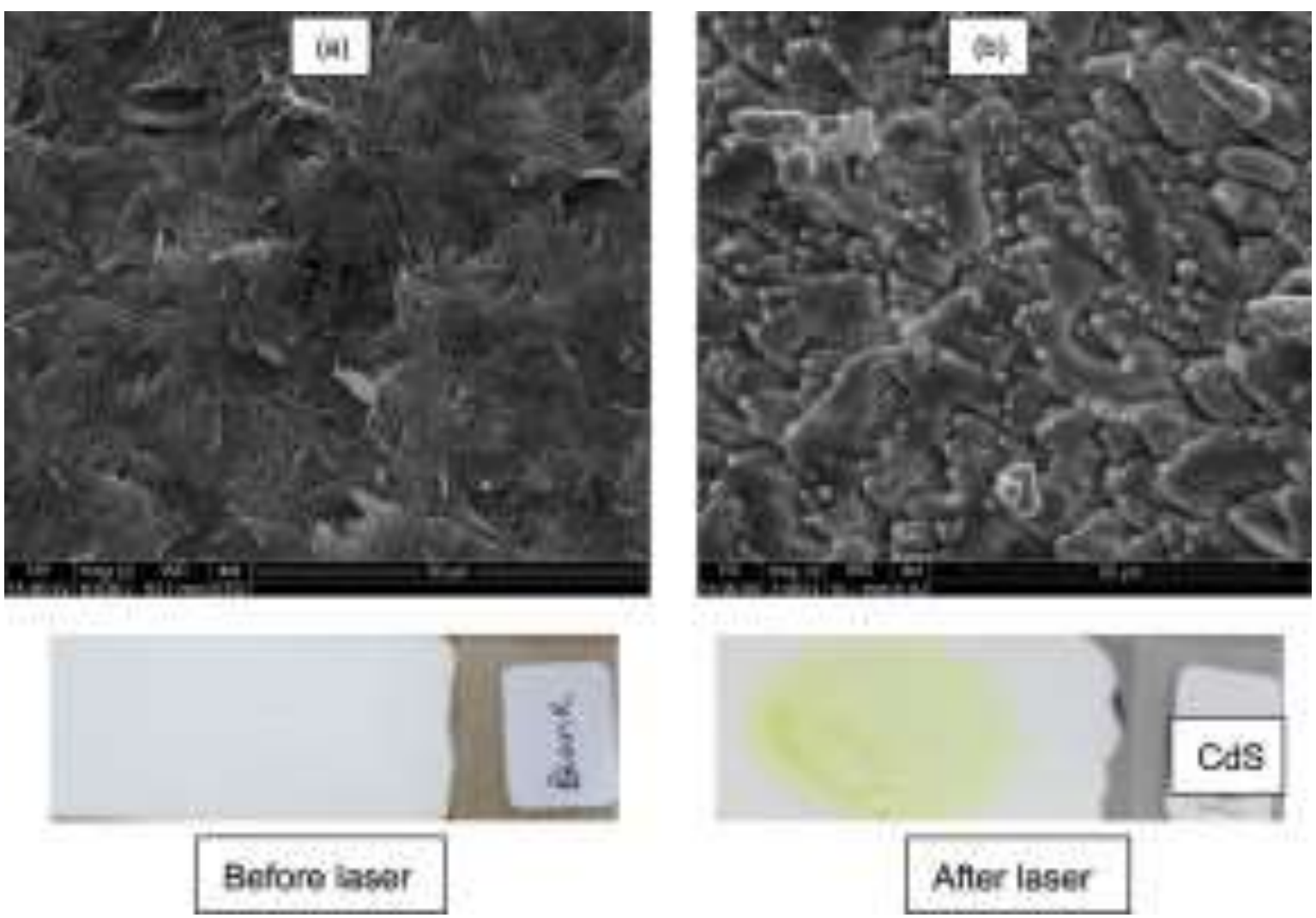

\section{Introduction}

Nanocrystalline materials have attracted much attention in recent years due to the change, at nanoscale, which occur when their crystallite radius becomes comparable to the exciton Bohr radius. Zinc sulphide and cadmium sulphide are among the widely studied II - VI group semiconductors because they show significant quantum confinement effects which 
influence their electrical and optical properties [1, 2]. They have found applications particularly in photovoltaic, photonic, and optoelectronic devices and sensors [3].

The syntheses of the II - VI group of nanoparticles have been achieved using different techniques which include thermolysis of a single-source precursor [4, 5], solventothermal [6], sonochemical [7], and colloidal precipitation [8]. However, most of these techniques are complex to perform with limited control over particle size [9].

Recently, interaction of radiation with matter has been reported as an efficient method for the synthesis of nanoparticles. Examples are microwave irradiation [10 - 13] as well as gamma ray irradiation in oil-in-water systems, the latter which was reported as an efficient method for the synthesis of mesoporous CdS semiconductor nanoparticles [14]. Production of nanoparticles by the use of laser irradiation either in gas or in vacuum has been explored during the last decade [15]. Laser irradiation of bulk molecules and subsequent decomposition into the nanoparticulate forms presents a simple route to the synthesis of nanoparticles. The laser activates the initial decomposition of the precursor and facilitates its conversion to the desired products under mild conditions. The entire procedure takes place in few hundreds of ns interaction time (s), since the nanoparticles are formed from the very first pulses of some nanoseconds (ns) duration [16]. A number of studies have exploited the use Laser irradiation of precursor materials for the synthesis of nanoparticles. For instance, syntheses of different noble metal nanoparticles by the ablation of metal surfaces immersed in liquid have been reported [17-20]. The surfactant surrounds each nanoparticle and prevents direct contact of the nanoparticles. The synthesis of II-VI compound semiconductor nanoparticles by the exposure of precursor compounds to laser 
radiation has been reported. Cadmium sulphide nanowires, and single crystalline three armed (nano tripods) CdS were prepared using a pulsed laser [21, 22]. Athanassiou et al. reported the formation of Zinc sulphide and cadmium sulphide nanocrystals, by the irradiation of $\mathrm{Zn} / \mathrm{Cd}$ thiolate precursors with UV laser pulses [23, 24]. The starting samples consist of polymers doped with the precursor complex which are decomposed after the absorption of UV light, resulting into the nanoparticles formation.

The precursor compounds used in this present study is the metal xanthate of zinc and cadmium. The decomposition of different metal xanthate precursors through thermolysis to produce metal sulphide nanoparticles have been reported [25]. Metal alkyl xanthates were used as the precursors in Lewis base alkylamine solvents. CdS nanoparticles capped with trin-octylphosphine oxide (TOPO) have been synthesised by a single-source route using cadmium ethyl xanthate as a precursor [26]. ZnS based thin films have been prepared by the decomposition of Zinc bis(O-ethyl xanthate) [27]. However, selection of the solvent and the choice of the temperature for thermal decomposition still require further research [25].

The chemistry of laser synthesis of nanoparticles from a precursor complex is derived from the fact that the absorption of an intensive laser pulse creates transitional or thermo-elastic stresses, which can lead to fragmentation. In particular, the energies of individual chemical bonds in organic molecules are around $400 \mathrm{~kJ} / \mathrm{mol}$ (e.g C-C, $E=347 \mathrm{~kJ} / \mathrm{mol} ; \mathrm{H}-\mathrm{H}, E=437$ $\mathrm{kJ} / \mathrm{mol} ; \mathrm{N}-\mathrm{N}, E=388 \mathrm{~kJ} / \mathrm{mol}$ ), which corresponds to the energy of near-ultraviolet (NUV) electromagnetic radiation. An NUV laser pulse with a sufficiently high energy density (i.e., intensity) will cause a large enough perturbation to break these chemical bonds [28, 29]. 
The method has been reported as an effective, flexible and efficient technique for preparing various types of high purity nanoparticles [9].

Here we present our research results on the synthesis of $\mathrm{ZnS}$ and $\mathrm{CdS}$ nanoparticles by nanosecond laser irradiation of zinc (II) and cadmium (II) ethyl xanthate. The effect of the length of time of the laser irradiation exposure on the sizes and the crystallinity of the prepared nanoparticles were studied. We report the surface morphology of the nanoparticles, the crystalline quality, and the optical properties. To the best of our knowledge, a time dependent studies of one-step, non-thermal, solid state reaction syntheses of ZnS and CdS nanoparticles via nanosecond laser irradiation from metal xanthate has not been reported. It is observed that the time of exposure to the laser irradiation has a significant effect on the properties of the nanoparticles synthesized.

\section{Experimental}

\subsection{Materials}

All the chemical reagents used for this study were obtained from commercial sources; they are of analytical grade and were used without further purification.

\subsection{Synthesis of potassium ethyl xanthate}

In a typical procedure (Zhang et al) [30], $0.1 \mathrm{~mol} \mathrm{KOH}$ powder was added into a beaker containing $0.11 \mathrm{~mol}$ absolute ethanol in a thermal bath at $50{ }^{\circ} \mathrm{C}$, followed by intensive agitation. After $45 \mathrm{~min}$, while still stirring, the solution was cooled down to room temperature and $0.10 \mathrm{~mol} \mathrm{CS}_{2}$ was added until a yellowish product precipitated. $100 \mathrm{~mL}$ of petroleum ether was added and stirring was continued for another $30 \mathrm{~min}$. The solid 
precipitate was filtered, rinsed with ethanol and dried at room temperature. Purification of the product was carried out by recrystallization using acetone and petroleum ether. The product was filtered, and dried in vacuum.

\subsection{Synthesis of cadmium and zinc ethyl xanthate}

The precursor complexes cadmium and zinc ethyl xanthate, $\left[\mathrm{M}\left(\mathrm{C}_{2} \mathrm{H}_{5} \mathrm{OCS}_{2}\right)_{2}\right](\mathrm{M}=\mathrm{Cd}, \mathrm{Zn})$, were prepared according to the procedure described as reported [31]. In a typical synthesis, $0.0025 \mathrm{~mol}$ of the metal salt (cadmium chloride/zinc chloride) was dissolved in $10 \mathrm{~mL}$ distilled water. A solution of potassium ethyl xanthate $0.8 \mathrm{~g}(0.01 \mathrm{~mol})$ in $10 \mathrm{~mL}$ distilled water was added and stirred for $45 \mathrm{~min}$. The white precipitate (pale yellow precipitate for cadmium complex) formed at the end of the reaction was filtered and rinsed several times with a mixture of ethanol-water, and dried under vacuum.

\subsection{Sample Preparation}

The microscope glass slides were cleaned by rinsing in diluted $\mathrm{HCl}$, and then sonicated in soap for $10 \mathrm{~min}$, followed by flooding with distilled water. Finally, they were rinsed in acetone and dried in the oven overnight. The samples for laser decomposition were prepared by dissolving $0.5 \mathrm{~g}$ of the respective complex in $10 \mathrm{~mL}$ chloroform and stirring vigorously to obtain a homogeneous solution. The substrates were casted manually on glass slides to produce thin homogeneous film after evaporation of the solvents. Drying of the samples was carried out in vacuo at room temperature. 


\subsection{Preparation of Nanoparticles}

The nanocrystals were formed by irradiating the samples with short, high-intensity laser pulses. Third-harmonic pulses at $355 \mathrm{~nm}$ from an Nd:YAG Q-switched nanosecond laser (EKSPLA NT342B-SH-10-AW) were used. The pulse repetition rate was $10 \mathrm{~Hz}$, while the pulse length and the pulse duration were $<0.13 \mathrm{~nm}$ and $\sim 4 \mathrm{~ns}$ respectively. The energy per pulse emanating from the laser source was determined as $11.00 \pm 0.26 \mathrm{~mJ}$ and decreased by 15 $16 \%$ before reaching the sample. Exposure times of $10 \mathrm{~min}$ and $30 \mathrm{~min}$ were used. The samples were positioned at the center of the reactor cell using microscope glass slides with dimensions $76 \mathrm{~mm} \times 26 \mathrm{~mm} \times 1 \mathrm{~mm}$ (LASEC). The laser beam was introduced into the chamber through a quartz window at an angle of $\sim 51^{\circ}$ with respect to the substrate normal to a diameter of $\sim 20 \mathrm{~mm}$ at the substrate surface.

\subsection{Sample characterizations}

The absorption measurements were carried out using a PerkinElmer Lambda 20 UV-vis spectrophotometer at room temperature. A PerkinElmer LS 55 luminescence spectrometer was used to measure the photoluminescence of the nanoparticles. A Quanta FEG 250 Environmental Scanning electron microscope (ESEM) was used to investigate the surface morphology of the nanoparticles. A thin gold layer was deposited to improve the electrical conductivity for better imaging. A JEOL 2100 TEM, fitted with a LaB $_{6}$ electron gun was used for the TEM imaging. Samples were suspended in DMSO, sonicated for 1 min and dispersed on carbon-coated grids. Analysis was done at $200 \mathrm{kV}$, and images were captured using a Gatan Ultrascan digital camera. The X-ray powder diffraction data were collected on a Röntgen PW3040/60 X'Pert Pro X-ray diffractometer using Ni-filtered Cu Ka radiation $(\lambda=$ 
$1.5405 \AA$ A) at room temperature. X'Pert HighScore Plus PW3212 software was used for the analysis and the phase identification was carried out using standard JCPDS.

\section{Results and discussion}

\subsection{Synthesis}

The synthetic procedures used for the sodium ethyl xanthate and the cadmium/zinc complexes followed the reported procedures in $[30,31]$. The results of the characterization are in accordance with literature reports. Schematic diagram of the laser induced decomposition chamber is shown

in scheme

1.

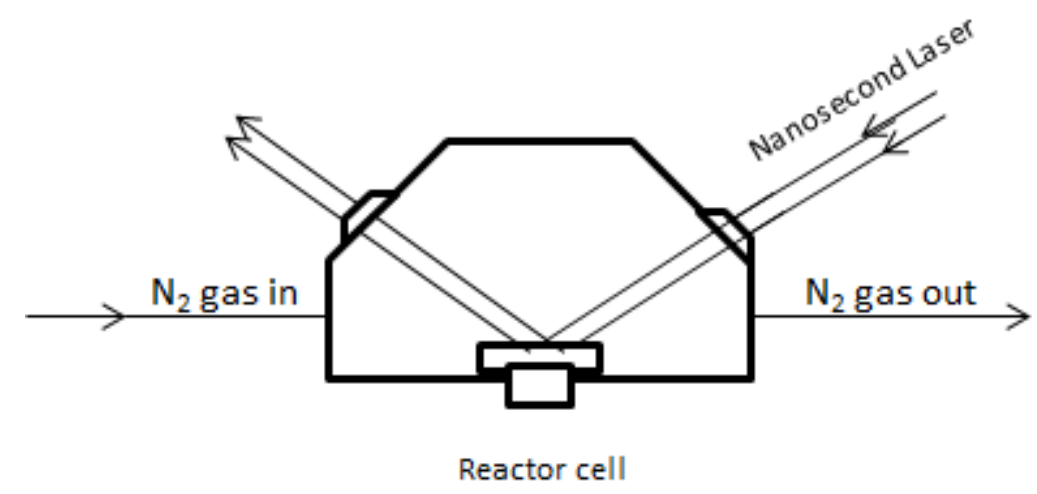

Scheme 1. Schematic diagram of the Laser set-up

\subsection{Optical properties}

The electronic properties of nanoparticles change significantly as the dimension of the particles become comparable or less than the Bohr radius of the exciton. This is because the lowest energy level of the exciton is now delocalized over the entire nanoparticle. The change is observed as a blue shift in the absorption spectra of the nanoparticle relative to the bulk compound. Figure 1 shows the UV absorption spectra for (A) ZnS and (B) CdS 

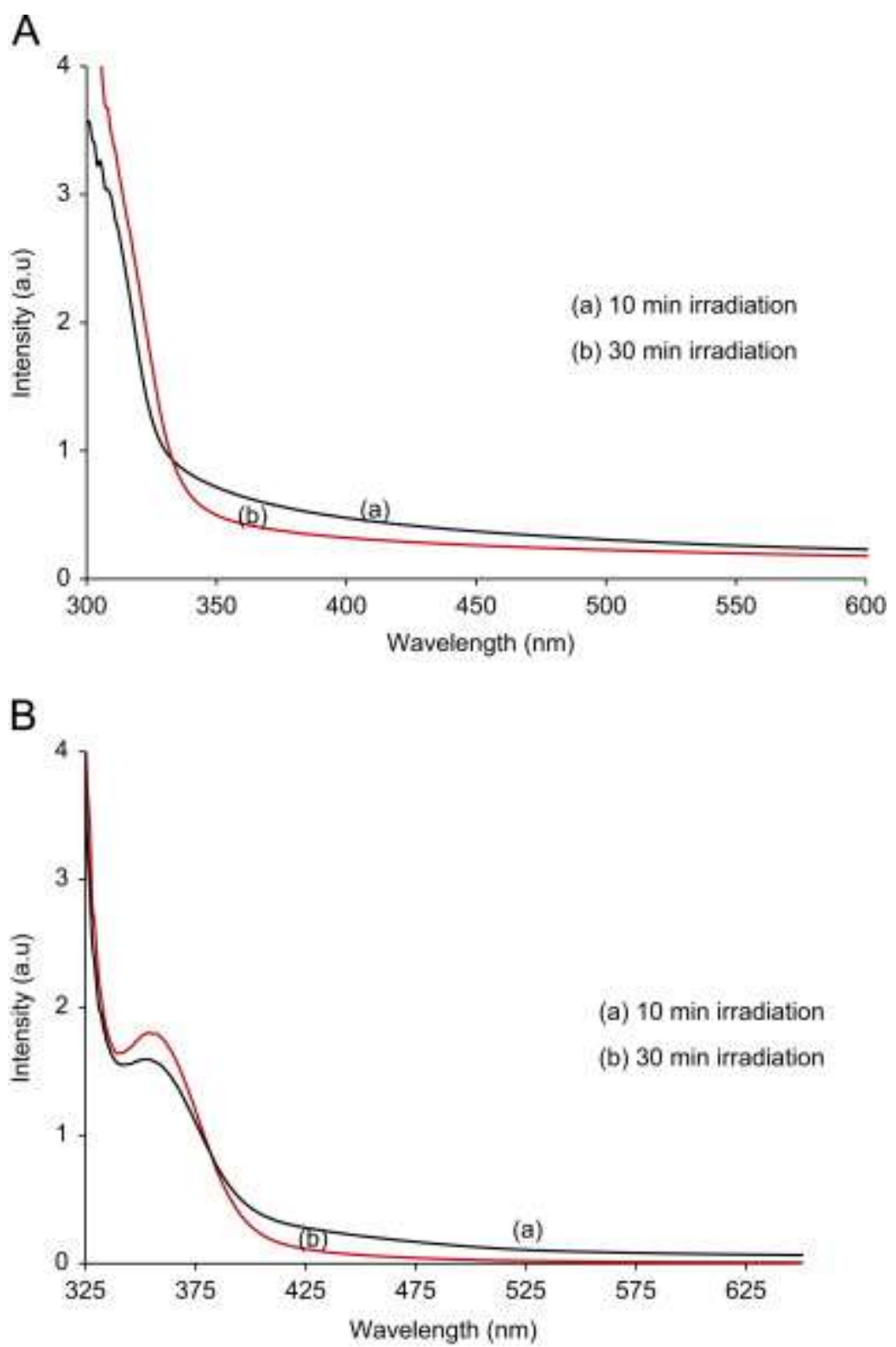

Fig. 1. : Absorption spectra of (A) ZnS, and (B) CdS nanoparticles obtained after (a) 10 min (black) and (b) $30 \mathrm{~min}$ (red) nanosecond laser irradiation

samples at $10 \mathrm{~min}$ and $30 \mathrm{~min}$ laser irradiation time respectively. All the spectra show a blue shift in the absorption spectra compared to the value of their respective bulk, ZnS (345 nm) [32] and CdS (512 $\mathrm{nm})$ [33]. The blue shift of the optical absorption spectra is associated with an increase in the band gap energy and indicates the formation of particles in the nanometer size regime [34]. 
The CdS nanoparticles exhibit the characteristic shoulder at around $360 \mathrm{~nm}$. This band has been observed around $266 \mathrm{~nm}$ in CdS nanoparticles prepared by the laser irradiation of a Cd complex embedded in a polymeric material, using the laser wavelength of 266 and $355 \mathrm{~nm}$ [16]. Under the same laser fluence, formation of nanocrystals at $266 \mathrm{~nm}$ was faster than at $355 \mathrm{~nm}$, due to the high absorption efficiency of the precursor at the wavelength of the former. The red shift of the excitonic peak (with respect to the peak at $266 \mathrm{~nm}$ ) observed in our study may be due to the fact that the precursor was not embedded in a confined matrices during the nucleation and growth of the nanocrystals. The formation of nanocrystals from unimolecular precursor by laser processing takes place after the first irradiation shot, followed by the melting of smaller nanocrystals [34]. This phenomenon results into the formation of larger nanocrystals. The absorption band in CdS nanocrystals is attributed to the first excitonic transition between the ground state and the single electron-hole pair state $\left(1 S_{3 / 2} 1\right.$ se) [36]

The photoluminescence (PL) spectrum of ZnS nanoparticles after $10 \mathrm{~min}$ of irradiation displays a broad emission spectrum between 360 and $610 \mathrm{~nm}$ with a peak at approximately $403 \mathrm{~nm}$, which shifted to approximately $426 \mathrm{~nm}$ after $30 \mathrm{~min}$ irradiation time, as shown in Figure 2(A). This emission band could be ascribed to a recombination of electrons at the sulphur vacancy donor level with holes trapped at the zinc vacancy acceptor level $[37,38]$. Within the band gap in the nanocrystals are energy states, which are produced due to 


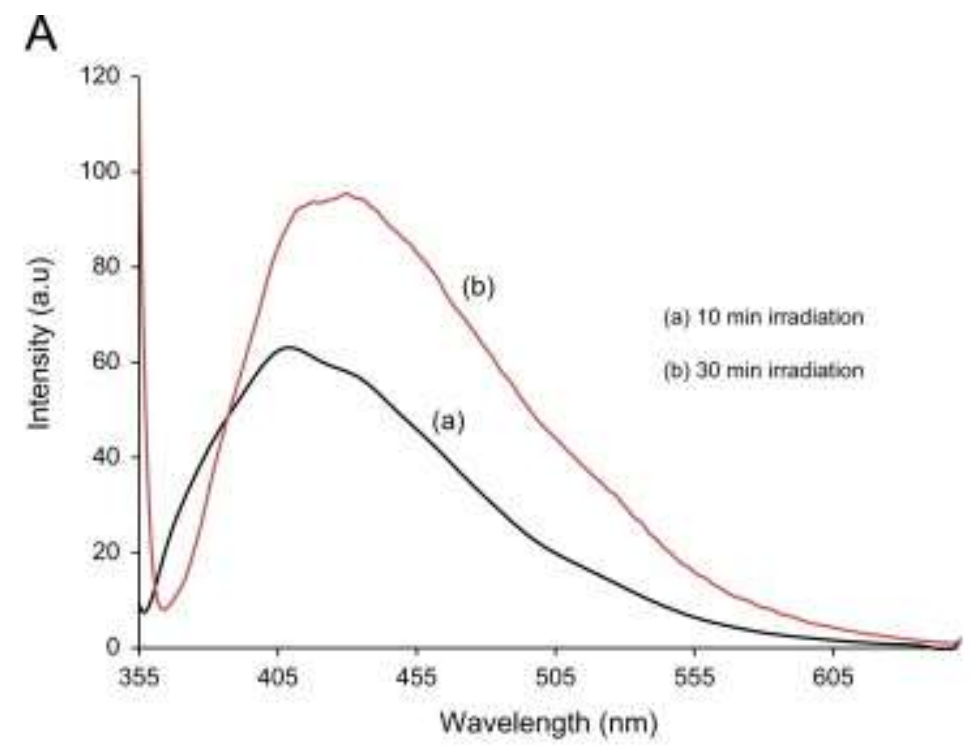

B

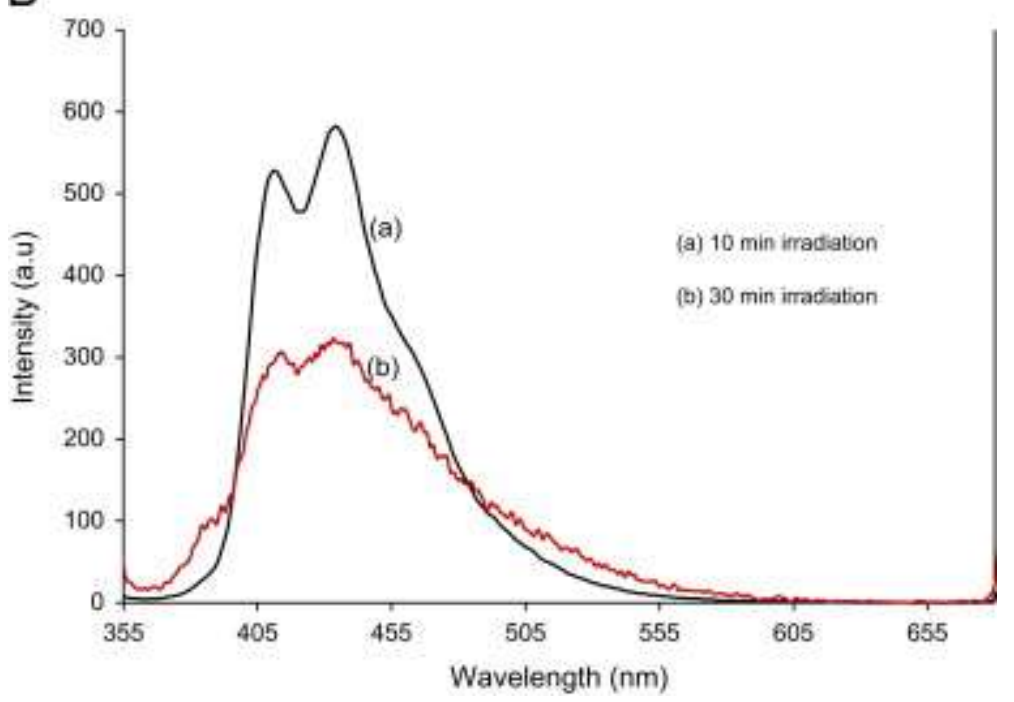

Fig. 2. : Photoluminescence spectra of (A) ZnS, and (B) CdS nanoparticles obtained after (a) 10 min (black) and (b) $30 \mathrm{~min}$ (red) nanosecond laser irradiation.

surface states or $\mathrm{Zn}^{2+}$ or $\mathrm{S}^{2-}$ ions. High energy photons excite the electrons from the valance band (VB) or $\mathrm{Zn}^{2+}$ levels, which reaches the conduction band (CB). The excited electrons first decay non-radiatively to surface-states, followed by a radiative decay to valance band. Thus, a photo of lower energy is emitted $[39,40]$. As the particle size increases, with increase in time of exposure, the valance band edge shifts upwards. Subsequently, the emitted photon has comparatively lower energy giving photoluminescence peak at higher wavelength. Chen 
et al. [41] has also reported PL peak of ZnS in this range, while Tamrakar et al [42] obtained the PL peak at $460 \mathrm{~nm}$ for uncapped ZnS nanoparticles, which then shifted up to $370 \mathrm{~nm}$ as capping concentration increased.

The broad and asymmetric emission of the samples may be due to size heterogeneity caused by the absence of the capping agent [43]. In CdS of nanoparticulate nature, the band edge emission and the surface defect emission lie in the wavelength range below and above $500 \mathrm{~nm}$ respectively. The PL spectra of CdS nanocrystals at both irradiation times (Figure 2B) exhibits broad and split peaks with maxima at $409 \mathrm{~nm}$ and $430 \mathrm{~nm}$, which is due to bandedge emission [44]. The splitting of PL peaks has been ascribed to different effects. Nisha et al. [45] observed a split at 405 and $426 \mathrm{~nm}$ for an $\mathrm{N}$-methyl aniline capped CdS nanoparticles synthesized at $120{ }^{\circ} \mathrm{C}$, and it was ascribed to the presence of different sized $\mathrm{CdS}$ nanoparticles. The split peaks observed at $460 \mathrm{~nm}$ and $475 \mathrm{~nm}$ by Pradhan et al. [46] was attributed to the presence of polygonal particles, while similar observations by Wang et al [47] was ascribed to the nucleation occurring simultaneously with the growth of some particles. Our result is similar to the observation by Nisha et al [45] and may be due to the presence of different CdS particle sizes. The reduced intensity of the CdS nanoparticles observed after $30 \mathrm{~min}$ irradiation could be due to the presence of defect. After the formation of the nanoparticles (10 $\mathrm{min})$, CdS can absorb the laser energy and have a defect by the prolonged laser irradiation. Photoluminescence properties usually depend on particle size, crystallinity or defect. 


\subsection{Morphological Properties}

The SEM images of the samples obtained before and after laser irradiation are presented in

Figure 3. The surface morphology after the laser irradiation showed obvious differences, indicating the formation of nanoparticles. The morphology of the zinc complex sample before laser irradiation appeared as a set of interlocked petal shaped particles (Figure 3(a)). The cadmium complex sample before laser irradiation showed set of spindles which formed aggregates of a well patterned mat (Figure 3(c)). After the laser irradiation, the mat-like morphology of the cadmium complex disappeared, giving rise to particles entrapped within
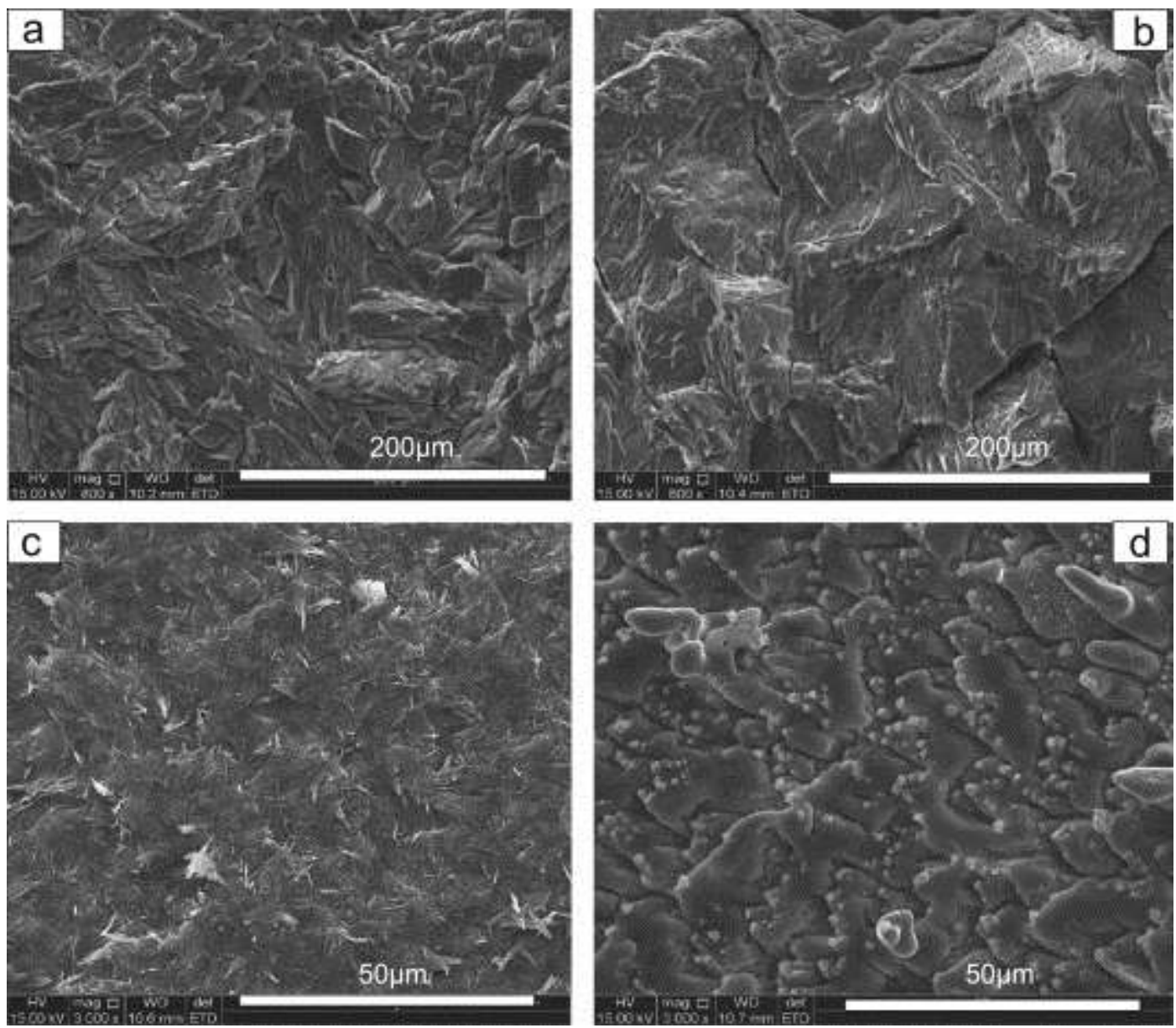

Fig. 3. : Surface SEM images (a) before (b) after nanosecond laser irradiation of Zn(II) bis(ethyl xanthate); and (c) before (d) after nanosecond laser irradiation of $\mathrm{Cd}(\mathrm{II})$ bis(ethyl xanthate) respectively. 
the surface (Figure 3(d)). The zinc complex sample showed no crystalline structures after the laser irradiation (Figure 3(b)). Similar observation has been reported in the synthesis of CdS and ZnS nanoparticles from cadmium thiolate and zinc thiolate precursors [24]. It was reported that in the absorption spectra of the prepared zinc sulphide and cadmium sulphide nanoparticles, peaks of the crystalline structures became evident in the absorption spectra after a few number of incident pulses in the $\mathrm{Cd}$-thiolate precursor. The $\mathrm{Zn}$-thiolate precursors required rather a prolonged irradiation [24]. In our case, although no crystalline structures were evident after 10 min laser irradiation of the $\mathrm{Zn}(\mathrm{II})$ bis(ethyl xanthate), the absorption spectra and the TEM images (Figure 4) of the samples indicate obvious formation of nanoparticles. The obtained $\mathrm{ZnS}$ nanopaticles after the laser irradiation of the $\mathrm{Zn}(\mathrm{II})$ bis(ethyl xanthate) were maybe too small to be observed under SEM or were very few and entrapped within the matrices of the bulk sample.

The TEM images of the samples obtained after $10 \mathrm{~min}$ of exposure to laser irradiation (Figure (4a)) showed that the ZnS nanoparticles are more crystalline than the CdS nanoparticles (Figure (4c)), but at an increased time of exposure to laser irradiation the crystallinity of the CdS increased (Figure (4d)). The CdS nanoparticles after 10 min irradiation could be estimated to an average size of $4.8 \mathrm{~nm}$ which grew into an oval shape of about 6.5 $\mathrm{nm}$ length and $3.5 \mathrm{~nm}$ widths after $30 \mathrm{~min}$ irradiation (Figure (4d)). It was difficult to estimate the size of the ZnS nanoparticles because the nanoparticles appeared to form aggregates with no crystalline edges when compared to the CdS sample. However, the crystallinity of the zinc sulphide nanoparticles reduced after $30 \mathrm{~min}$ laser exposure as observed in the diminished lattice fringes (Figure 4(b)). In our previous study [48], it was observed that nanoparticles prepared in the absence of a surfactant molecule formed a set 

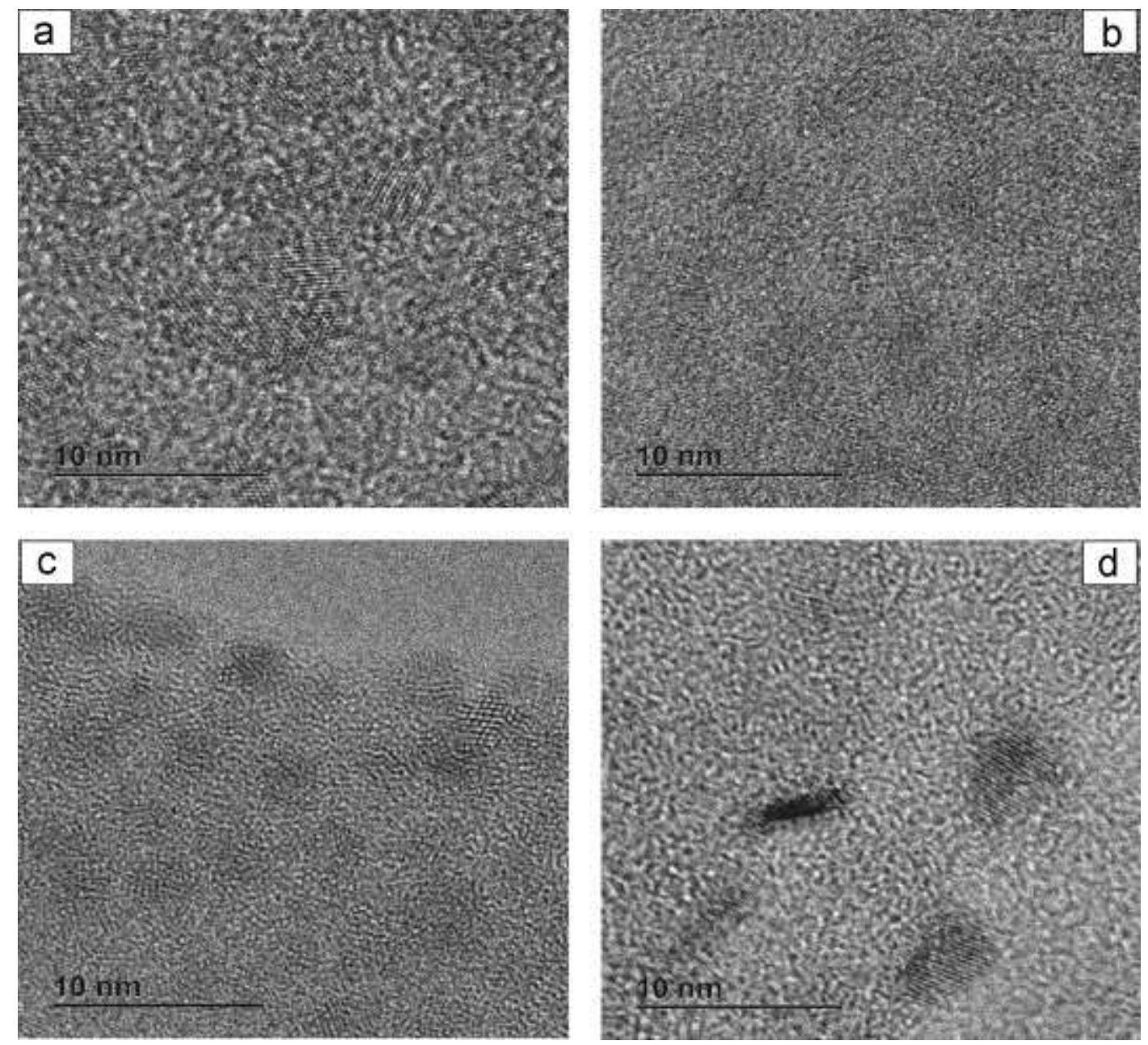

Fig. 4. : TEM images of (a) ZnS after 10 min laser irradiation, (b) ZnS after 30 min laser irradiation, (c) CdS after 10 min laser irradiation (b) CdS after 30 min laser irradiation. The ZnS sample after 10 min and CdS after 30 min show obvious lattice fringes which confirm the crystallinity of the nanoparticles.

of aggregates which were absent in the nanoparticles obtained in the presence of capping/surfactant molecules. It is therefore possible that in this present study, the obtained zinc sulphide nanoparticles lacked the benefits such as facial separation between nucleation and growth stages, the control of growth parameters and protection of the nanoparticles from agglomeration, which are associated with the nanoparticles obtained in a surfactant medium. Agglomerates are usually obtained due to the high surface reactivity of nanoparticles. 


\section{Conclusion}

Cadmium sulphide and zinc sulphide nanoparticles have been successfully prepared under nanosecond laser irradiation employing zinc and cadmium xanthate as precursor molecules, at room temperature. The cadmium sulphide was obtained as uniform, fine and crystalline particles, while the zinc sulphide formed crystalline aggregates. It was found that the time of exposure plays an important role in controlling the morphology of CdS nano-crystals. The optical spectra showed a red shift, indicating size increments, as the time of exposure to the laser irradiation increased from $10 \mathrm{~min}$ to $30 \mathrm{~min}$. Compared with the other synthesis methods, this method is much simple and faster.

\section{Acknowledgments}

This work was supported by National Research Foundation (NRF), South Africa, and North West University, Potchefstroom, South Africa. The authors are grateful to Dr Anine Jordaan of Laboratory for Electron Microscopy Northwest University for SEM analyses, and Dr James Wesley-Smith of the National Centre for Nanostructured Materials, CSIR South Africa for TEM analyses.

\section{References}

[1] N.V. Hullavarad, S.S. Hullavarad, P.C. Karulkar, J. Nanosci. Nanotechnol. 8 (2008) 3272

[2] S. Tiwari, S.Tiwari, Cryst. Res. Technol. 41 (2006) 78

[3] M. Sanz, M. Lopez-Arias, J.F. Marco, R. de Nalda, S. Amoruso, G. Ausanio, S. Lettieri, R. Bruzzese, X. Wang, M. Castillejo, J. Phys. Chem. C 115 (2011) 3203.

[4] B. Ludolph, M.A. Malik, P. O’Brien, N. Revaprasadu, Chem. Commun. (1998) 1849

[5] J.C. Bruce, N. Revaprasadu, K.R. Koch, New J. Chem. 31 (2007) 1647 
[6] S. Yu, Y. Wu, J. Yang, Z. Han, Y. Xie, Y. Qian, X. Liu, Chem. Mater. 9 (1998) 2309

[7] M.L. Breen, A.D. Dinsmore, R.H. Pink, S.B. Qadri, B.R. Ratna, Langmuir 17 (2001) 903

[8] W. Chen, Z. Liu, Z. Wang, Solid State Commun. 100 (1996) 101

[9] R. Zamiri, A. Zakaria, H.A. Ahangar, M. Darroudi, A.K. Zak, G.P.C. Drummen, J. Alloys Compd. 516 (2012) 41

[10] W. Jian, J. Zhuang, D. Zhang, J. Dai, W. Yang, Y. Bai, Mater. Chem. Phys. 99 (2006) 494

[11] H. Yang, C. Huang, X. Li, R. Shi, K. Zhang, Mater. Chem. Phys. 90 (2005) 155

[12] Y. Zhao, J.-M. Hong, J.-J. Zhu, J. Crystal Growth 270 (2004) 438

[13] F.A. La Porta, M.M. Ferrer, Y.V.B. de Santana, C.W. Raubach, V.M. Longo, J.R. Sambrano, E. Longo, J. Andrés, M.S. Li, J. A. Varela, J. Alloys Compd. 556 (2013) 153

[14] J. Chen, X. Wang, Z. Zhang, Mater. lett. 62 (2008) 787.

[15] P.V. Kazakevich, A.V. Simakin, V.V. Voronov, G.A. Shafeev. App. Surf. Scie. 252 (2006) 4373

[16] D. Fragouli, A. M. Laera, P. P. Pompa, G. Caputo, V. Resta, M. Allione, L. Tapfer, R. Cingolani, A. Athanassiou, Microelectron. Eng. 86 (2009) 816.

[17] A. Fojtik, A. Henglein, Ber. Bunsen-Ges. Phys. Chem. 97 (1993) 252.

[18] M. S. Sibbald, G. Chumanov, T. M. Cotton, J. Phys. Chem. 100 (1996) 4672.

[19] M. S. Yeh, Y. S. Yang, Y. P. Lee, H. F Lee, Y. H. Yeh, C. S. Yeh, J. Phys. Chem. B 103 (1999) 6851.

[20] F. Mafune', J. Kohno, Y.Takeda, Tamotsu Kondow, J. Phys. Chem. B 104 (2000) 9111.

[21] N. Kuthirummal, J. Reppert, B. Dihel, A.M. Rao, Appl. Opt. 48 (2009) 2842.

[22] D. C. Bell, O. Hayden, A. B. Greytak, C.M. Lieber, Microsc. Microanal. 10 (2004) 1.

[23] G. Bounos, A.Athanassiou, D. Anglos, S. Georgiou, C. Fotakis, J. Phys. Chem. B 108 (2004) 7052.

[24 ] A. Athanassiou, L. Blasi, M. De Giorgi, G. Caputo, D. Fragouli, E. Tsiranidou, A.M. Laera, L. Tapfer, R. Cingolani, Polym. Compos. 31 (2010) 1075

[25] W-M. Zhang, Z-X. Sun, W. Hao, D-W. Su, D. J. Vaughan, Mater. Res. Bull. 46 (2011) 22682266.

[26] Y. K. Jung, J. Kim, J-K. Lee, J. Am. Chem. Soc. 132 (2010) 178. 
[27] D. Barreca, E. Tondello, D. Lydon, T.R. Spalding, M. Fabrizio, Chem. Vap. Dep. 9 (2003) 93.

[28] M. Frumar, B. Frumarova, P. Nemec, T. Wagner, J. Jedelsky, M. Hrdlicka, J. Non-Crystal. Solids 352 (2006) 544

[29] P. Nemec, M. Frumar, J. Optoelectron. Adv. Mat. 5 (2003) 1047

[30] W-M. Zhang, Z-X. Sun, W. Hao, D-W. Su, D. J. Vaughan, Mate. Res. Bull. 46 (2011) 2266

[31] P.S. Nair, T. Radhakrishnan, N. Revaprasadu, G. Kolawole, P. O’Brien, J. Mater. Chem. 12 (2002) 2722

[32] E.K. Goharshadi, R. Mehrkhah, P. Nancarrow, Mater. Sci. Semicond. Process. 16 (2013) 356

[33] L. Xu, Y. Su, D. Cai, Y. Chen, Y. Feng, Mater. Lett. 60 (2006) 1420

[34] M. Pattabi, B.S. Amma, Sol. Energy Mater. Sol. Cells 90 (2006) 2377

[35] V. Resta, A. M. Laera, A. Camposeo,E. Piscopiello, L. Persano, D. Pisignano, L. Tapfer, J. Phys. Chem. C 116 (2012) 25119.

[36] H. M. Schmidt, H.Weller, Chem. Phys. Lett. 129 (1986) 615

[37] S. Sapra, A. Prakash, A. Ghangrekar, N. Periasamy, D.D. Sarma, J. Phys. Chem. B 109 (2005) 1663

[38] M.V.R. Krishna, R.A. Friesner, J. Chem. Phys.95 (1991) 8309

[39] A.K. Shahi, B.K. Pandey, R.K. Swarnkar, R. Gopal, Appl. Surf. Sci. 257 (2011) 9846.

[40] S.K. Mehta, S. Kumar, M. Gradzielski, J. Colloid Interface Sci. 360 (2011) 497.

[41] L. Chen, J. Zhang, Y. Luo, S. Lu, X. Wang, Appl. Phys. Lett. 84(2004) 112

[42] R. Tamrakar, M. Ramrakhiani, and B.P. Chandra, Nanoscience J. 2 (2008) 12.

(43] G. Murugadoss, Particuology 11 (2013) 566

[44] W.S. Chae, J.H. Ko, I.W. Hwang, Y.R. Kim, Chem. Phys. Lett. 365 (2002) 49 
[45] K.D. Nisha, M. Navaneethan, B. Dhanalakshmi, Y. Hayakawa, S. Ponnusamy, C. Muthamizhchelvan, Opt. Mater. 35 (2013) 1652

[46] N. Pradhan, B. Katz, S. Efrima, J. Phys. Chem. B 107 (2003) 13843

[47] Q. Wang, D. Pan, S. Jiang, X. Li, L. An, B. Jiang, Chem. Eur. J. 11 (2005) 3843

[48] D.C. Onwudiwe, C.A Strydom, O.S Oluwatobi, New J. Chem. 37 (2013) 834 